\title{
The Experimental Investigation of The performance of Phosphor Coating
}

\author{
J.G Alotaibi ${ }^{a}$, Ayedh Alajmi ${ }^{b}$, Ahmad $S M$ Alotaibi ${ }^{c}$ \\ ${ }^{\mathrm{a}, \mathrm{b}}$ Department of Automotive and Marine Technology The public Authority for \\ Applied Education and Training, Collage of Technological Studies, Kuwait \\ ${ }^{c}$ Operation and maintenance Department, Ministry of electricity and water, \\ Kuwait
}

Abstract- Different screen printing parameters have a distinct effect on the quality of coating that is created. This experimental work looked to measure these parameters. It is divided into two parts where the first part measured the surface wettability of various substrates Wettability measurement was performed through measuring the contact angle between the droplet and the substrate surface. Varying the UV exposure time by putting substrate in the UV ozone cleaner has a considerable effect on the wettability of the surface. Water was supplied to a PEDOT: PSS substrate via Fibro DAT 1100 which is attached to a camera and a computer for capturing and analyzing coatings for the spread of a water droplet on the substrate surface. From these analyses, the main aim is to measure the contact angle between the droplet and the substrate surface which indicates the surface wettability. Some factors may affect the wettability of the surface such as varying the UV time and varying the hold time. As a result, increasing these times will decrease the contact angle where a small contact angle indicates a favorable wettability. The second part measured the electrical resistance and transparency of the printed ink after various printing experiments such as implementing UV treatment, changing the ink and changing the mesh ruling. The Rheometer device was used to test the properties of the specimen. Where this device can be used to measure the properties of the specimen such as the shear modules, in this paper the plate was used to effect on the specimens, where different readings are recorded for these specimens. Also, 24 samples were prepared and the electrical resistance for these samples was measured in this paper. As a result, the electrical resistance for treated samples was greater than that for untreated samples; the electrical resistance of Elentan ink was greater than that for PEDOT at the same conditions. Changing the screen size from (61-64) to (40-100) increase the value of resistance at the same conditions. For the transparency measurements the Elentan ink had a higher transparency than PEDOT ink. The paper also carried out an analysis for the relation between the thickness of the phosphor layer and the lamp output, where the relation was inversely proportion.

Keywords-PEDOT; ELNTAS; IPA; UV; Phosphor; electroluminescent

\section{INTRODUCTION}

Electroluminescence (EL) is a relatively low power display technology that can be manufactured by printing and can be used on both ridged and flexible substrates. Predominantly it has been used for signage and advertising, however, in the majority of these applications a single phosphor is used to provide a single color output.
EL in application had been around in form of electroluminescent wire (or EL wire) since it was invented in 1936 by Georges Destiau, a French physicist [1]. EL materials were available in sheet or wire forms, the wire form being widely accessed encased in glass or ceramic. This limited the usefulness of EL wires owing to the non-malleable nature of both glass and ceramic, due to which these encasing materials were replaced by pliable plastic coating which was waterproof as well. Electroluminescence as a principle is similar to that of a laser in terms of the production of photons when an excited substance returns to its ground state, except that in case of EL the operating energy is quite low and the light produced is rather incoherent [2]. EL devices include light emitting diodes (LEDs) which produce light which produce light through application of light into a doped p-n junction, and EL displays (ELDs) that are used for displaying graphics, texts and computer coatings as matrix-addressed devices. The basic structure of an ELD involves a minimum of six layers consisting of a base plate, conductor, insulator, phosphors layer and additional insulators and conductors.

Kobayashi et al. (2000) worked on developing a new RGB display device which was multi- colored and made of a lightemitting polymer (LEP) poly (para-phenylene vinylene) (PPV) which was spin-coated by poly (di-octyl fluorene) (F8). This system was successfully applied in producing EL layer patterns on a thing film transistor substrate through an inkjet process which produced the required RGB display output. Accessing relevant patent works also should be a part of this method as necessary technical background can be established. An invention by Kitazume et al [3] of an organic EL device is one such source. The patent featured a substrate with a first electrode over it which is separated by a partition wall, the latter having a second partition wall located on its inner side an organic luminous layer is included in the organic luminescent media layer on which a second electrode is placed facing the first electrode. according to Lee and Lee (2011) [4] proposed fabrication method for cholesteric liquid crystal capsule display where the capsules for the RGB display were separated in their rendering of sub pixels by a width of $200 \mu \mathrm{m}$. On a similar line of work, Quites et al. (2014) worked on polymer LEDs on their white emission by combining two materials with different emission properties on a single component system. It involved a PFOFP en-diode for the EL emission which was absorbed by a red emitter component MDMO-PPV [4]. Further insight can be obtained from a proper review of their paper. Similarly more such works need to be reviewed for establishing a 
comprehensive framework of the current project work in terms of screen printing process for RGB panels, colour filtering of their output, selection of EL diodes and substrates and then their suitable encapsulation. Additionally, a market study of EL consumer products might be required to add value and useful contribution into the framework on EL printing and fabrication of devices.

The project method also needs to study available EL devices and technologies in order to gain suitable insights pertaining to RGB display panel outputs. There are different screen printing parameters have a distinct effect on the quality of coating that is created. In this paper, the experimental work looked to measure screen printing parameters. It is divided into two parts where the first part measured the surface wettability of various substrates Wettability measurement will performed by measuring the contact angle between the droplet and the substrate surface. Varying the UV exposure time by putting substrate in the UV ozone cleaner has a considerable effect on the wettability of the surface. In addition, the second part will be measuring the electrical resistance and transparency of the printed ink after various printing experiments such as; implementing UV treatment, changing the ink as well as changing the mesh ruling.

\section{A. - Methodology}

The methodology in this paper is based on experimental work to measure screen printing parameters in order to investigate in quality of the coating by measuring the surface wettability of various substrates Wettability measurement as well as measuring the electrical resistance and transparency of the printed ink after various printing experiments.

\section{CONTACT ANGLE MEASUREMENTS}

Contact angle measurements are one of the experimental tools that are used to understand the wetting between a substrate and a liquid. A liquid property to adhere and the sample absorption of liquid if they are porous like PET, PP, and ITO are very significant in different aspects during the manufacturing and converting processes and also in the end-use fields of converted sample. Also, quality of printing process is affected by different factors such as the ink type, the contact angle, wettability and the surface energy [5]. Where the contact angles can be affected by absorption properties of a substrate, but not all substrates absorb water. Polypropylene, PET, ITO all cause water to bead on the surface. The wetting behavior between a substrate and a liquid depends on the relationship between the substrate surface energy and the liquid surface energy. The nature of relationship between these energies is not simple, and also the proposed mathematical models for wettability, adhesion as well as absorption are outside the scope of this project, but already well documented as in[6] and [7] they measured the contact angle using different nozzles according to the hydrostatic pressure. In several cases, the angle subtended between the edge of the droplet at rest a substrate, is used to predict and understand the end-use results of a printing or coating processing as well as sorptive application because it's important to specify the characteristics of wettability or a substrate has low surface energy, and ink may not adhere to the substrate because they effect on the printing quality. [8]. In this experiment, the dynamic contact angle which refers to the surface wettability for the samples will be measured using Fibro DAT 1100 device under two conditions; UV exposure time by putting substrate in the UV ozone cleaner, because the UV/ozone is usually used to treat the surfaces, where the both of surface tension and the base component increased, then the change decayed in surface tension related to $\mathrm{UV} / \mathrm{O}_{3}$ treatment after the treatment. The difference is small, when treated and untreated surface are used. Also, many substrates behave under different decayed rate as shown by [9].

\section{A. - Equipment's and Material}

This test method aims to measure the dynamic contact angle of a certain test liquid which is in contact with a flat specimen. Fibro DAT 1100was used to measure the dynamic contact angle in this work is the Fibro DAT 1100. This equipment enables an automatic and detailed analysis at full video speed of the contact between a droplet of a liquid and substrate surface. The resultant time-dependent wetting and absorption response associates to several experienced production problems such as in adhesion, sizing, surface coating and printing. In this experiment it is used for printing issues. A CCD camera will be used to captures coatings of the drop where a computer will measure the dimensions of the drop. The De-ionized water was used throughout the experiments. The substrate material used in this experiment is PET Polyethylene terephthalate. It is a thermoplastic polymer used in different industries such as in thermoforming applications, liquid containers, food, and beverage. This material is considered as an excellent moisture and water barrier. By obtaining these values, the absorption can be exactly specified and a well conclusion can be generated about the ink situation. Figure below presents the measured variables.

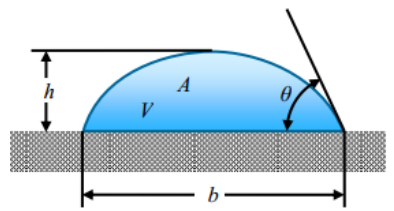

Figure 1: Drawing of the Drop on the Surface Throughout the Wetting Process.

\section{B. - Test procedure}

The test procedure will be as followed:

- A water drop with a known volume is dispensed onto the PET surface through a syringe using the fibro DAT 1100.

- The video camera captures coatings of the water drop in contact with the PET substrate at regular time intervals.

The computer is then used for coating analysis to contact angle directly from the coating.

- The contact angle measurements were carried out on three different days as followed:

- On the first day, the test procedure was applied to one PET specimen to prepare it for next steps. 
- In the second day, the test procedure was applied to one PET specimen at different UV and hold times under UV to see the effect of that on the contact angle.

- On the third day, the test procedure was applied on three PET specimens at the same time to indicate the average value.

At this experiment the effect of UV on the contact angle was measured.

\section{C. - Test Results}

The obtained results from following the above procedure are presented in this section. These results show the change in the contact angle of the liquid droplet as a function of time. The first results aim to discover the effect of varying the UV time on the contact angle measurements, when contact angle increases when the Untreated specimen are used where the results shows the data goes to stability after one second approximately. However to discover the effect of varying the time of Hold time on the contact angle measurements and. Figure .2 shows this effect where the contact angle increases with increasing the holding time where the holding time in minutes .

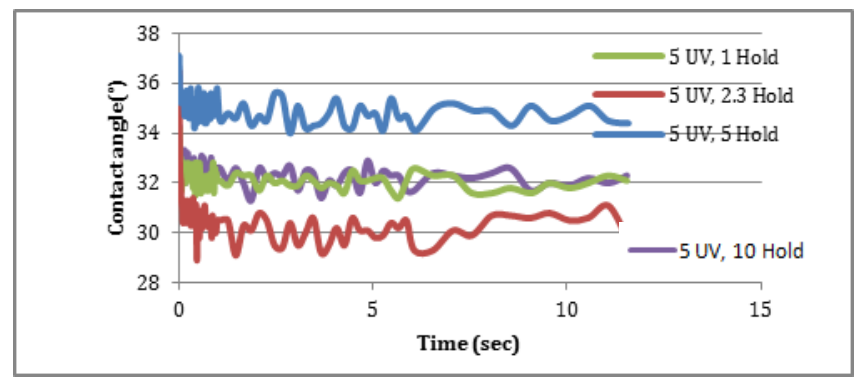

Figure 2: Results with Varying the Hold time.

In Figure. 4 The experiment were performed to measure the contact angle for three PET substrates at different UV hold times in second. The results were obtained for each specimen separately and then combined in one and then combined in one figure which presents the average value from the three specimens. This figure is presented below.

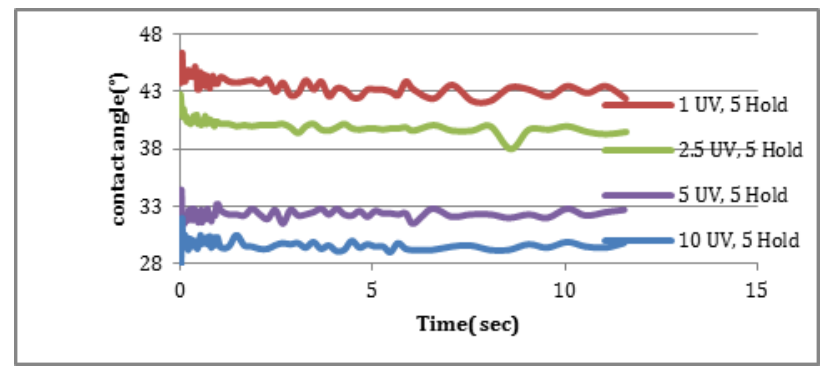

Figure 3: The Average Contact Angle Measurement for Three PET Specimens.

As shown in the figure the contact angle affected by the holding time where the maximum recorded angle about $45^{\circ}$ and the minimum about $28^{\circ}$, the stability of the results shows the time to achieve the stability is long.

\section{CONDUCTIVITY AND TRANSPARENCY MEASUREMENTS}

Conductivity of the inks was performed by measuring the resistance of a variety of line widths printed onto the substrate. The lines would vary in resistance in accordance to Ohm's Law.

A control sample with UV treatment applied was used, while the others had different ink types and different the screen sizes.

\section{A. Equipment's and Materials}

A multi meter was used in this experiment to measure the electrical resistance of the printed inks. The transparency was measured using a Viptronic Vipdens 620 transmission densitometer. It is used to measure the percentage of light transmission through the samples. In this experiment two types of inks will be; PEDOT: PSS and Elentan inks.

\section{B. Test procedure and measurements}

In the previous experiment different transparent samples were exposed to UV treatment while some of them remain without treatment. Two measurements were taken; the electrical resistance and the transparency

The purpose of these measurements was to correlate various screen printing parameters against the electrical resistance and transparency these parameters are;

- UV surface treatment, the results was obtained once with applying UV treatment and once without implementing it.

- The type of Ink.

- Two types of inks will be used to measure their effect on the wanted results.

- $\quad$ The mesh ruling which is described the threads per $\mathrm{cm}$ and the thickness of the thread.

\section{E. ELECTRICAL RESISTANCE MEASUREMENTS}

The first type of results was for electrical resistance with and without UV treatment as shown in the following.

\section{A. - With uv treatment}

The first figure below shows the variation of electrical resistance measures versus the ink thickness measured in ( $\mathrm{mm})$ with applying UV treatment. From the figure, the Elentan ink with size 40-100 has the highest electrical resistance According to Ohms law. Also the figure shows that Elentan ink has higher electrical resistance than PEDOT in both mesh ruling.

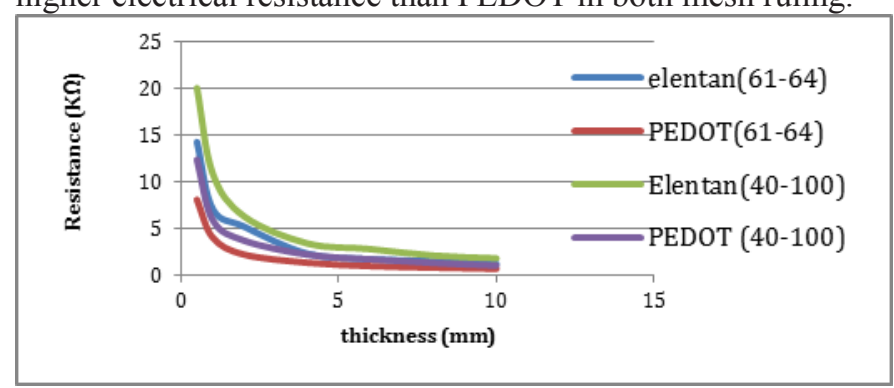

Figure 4: Resistance of Printed Conductive Lines onto UV Treated Substrate Plotted Against Ink Film Thickness 


\section{B. - With no UV treatment}

The second figure below shows the variation of electrical resistance versus the ink thickness without applying UV treatment. As with the UV treated samples, the Elentan ink with size 40-100 has the highest electrical resistance. Also the figure shows that Elentan ink has higher electrical resistance than PEDOT in both screen sizes. But when these results are compared to the obtained results in the previous figure it was concluded that applying UV treatment increased the electrical resistance of the sample.

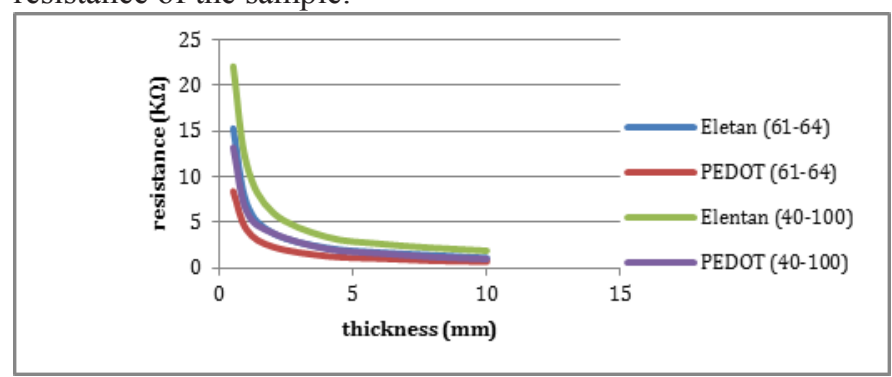

Figure 5: Resistance of Printed Conductive Lines onto Non-UV Treated Substrate Plotted Against Ink Film Thickness.

\section{C. - With varying the ink type}

\section{1) Using Pedot ink}

The following figure presents the results with using PEDOT as the ink. from the figure the highest electrical resistance obtained for PEDOT that was by using a mesh ruling of 40100 without UV treatment.

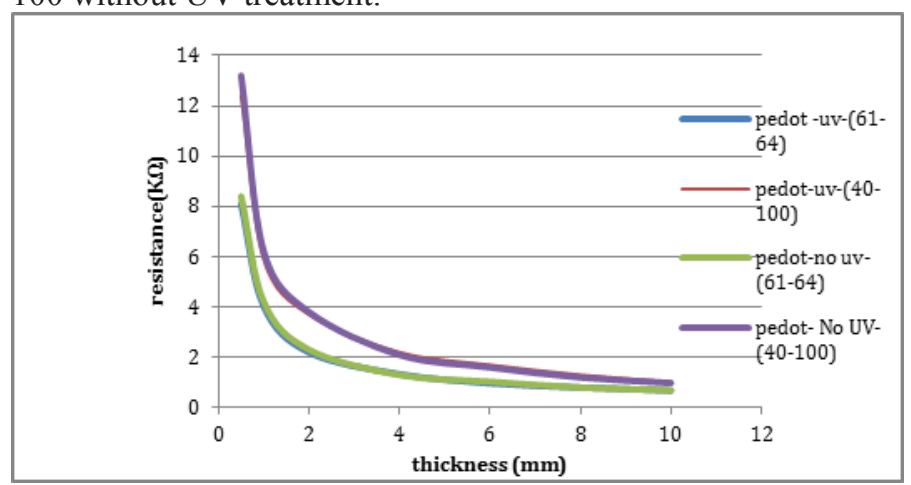

Figure 6: resistance with using PEDOT ink.

2) - Using Elentan ink

With changing the ink type to Elentan, then the following figure presents the results with using Elentan the ink. From the figure that the highest resistance obtained for electrical resistance was for Elentan that has a mesh ruling of 40-100 without UV treatment. When it's compared to the PEDOT results it was found that the electrical resistance of Elentan is more than that for PEDOT at the same conditions.

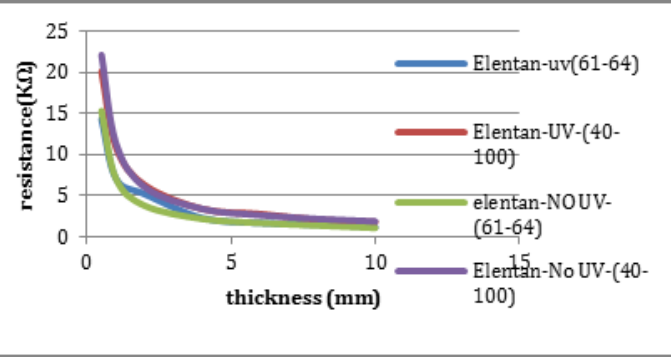

Figure 7: resistance with using Elentan ink. Varying the screen size

The overall results shows PEDOT: PSS was more conductive than Elentan ink, 61-64 Mesh ruling gave more conductive results than 40-100, also UV treatment seemed to have no effect on the conductivity

\section{F. TRANSPARENCY MEASUREMENTS}

The second results were for the transparency of the two inks at different conditions. The plot below presents these results. The results show that Elentan ink with UV treatment has the highest transparency, also by comparing the same ink and same treatment with the two different mesh rulings. 61-64 mesh ruling gave better transparency than the 40-100 mesh ruling.

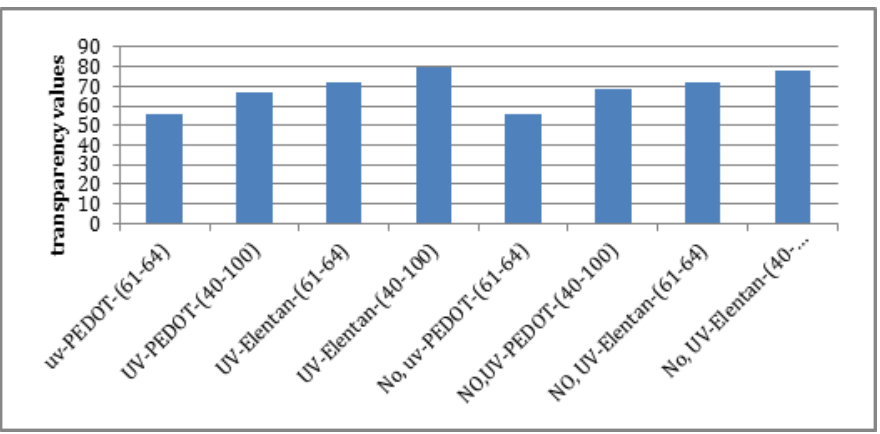

Figure 8: transparency measurements.

\section{ELECTROLUMINESCENT DESIGN}

The light Emitting capacitor is used to produce a light when the crystals of phosphor are excited by electric current [12], this technology are used by different applications like the LCDs, control panels, cell phones and architecture applications [13 ]. In this experiment the following capacitor was structured

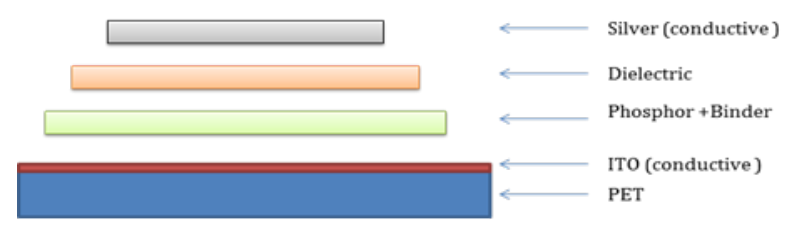

Figure.10: the structure of the Capacitor

Where the electron moves from the positive side to the negative side where the silver is the positive side and the ITO 
is the negative, the movement of electrons causes phosphor crystal excitation. Different factors effect on the capacitance like the type of materials and their relative permittivity, distance between the two conductive materials according to the following equation:

$$
\mathrm{C}=\varepsilon_{o} \frac{A}{d}
$$

Where

$\mathrm{C}$ : the capacitance

$\varepsilon_{o}$ : The relative permittivity

A: cross sectional area

D: the distance between the conductive

As shown, the relation between the capacitance and the distance is inversely proportion which means when the distance increases the capacitance will decrease.

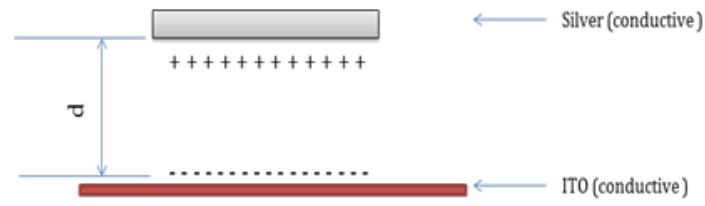

Figure.11: poles of the capacitor

In this research, 8 experiments are conducted using different thicknesses. With taken into consideration the gap when the thin layer is connected is $1 \mathrm{~mm}$ and the gap when thick layer is connected is $1.5 \mathrm{~mm}$. This means the thick layer required more excitation energy to move the electron cross the gap. The layers are fixed to gather to design a capacitor as shown in the following figure; the thickness of the capacitor is variable, so the capacitance is varying according to the distance between the two conductive. Then the lump output $\left(\mathrm{Cd} / \mathrm{m}^{2}\right)$ was measured

The lamp brightness at each thickness are shown in the following figure where the maximum lamp output was measured for the 1 thick layer with value $174 \mathrm{Cd} / \mathrm{m}^{2}$, and the minimum value was recorded for 4 thin layers with value 62.5 $174 \mathrm{Cd} / \mathrm{m}^{2}$.

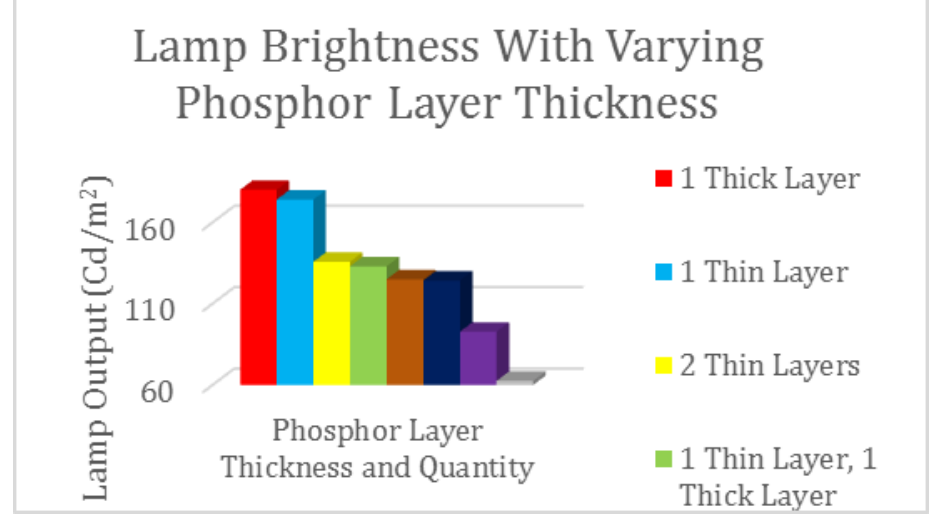

Figure.12: Lamp Brightness with Varying Phosphor Layer Thickness

In order to find a good fitting between the layer thickness and the lamp output the following curve was plotted as shown in the following curve, the average values where plotted for different readings where 8 trials was taken for each experiment

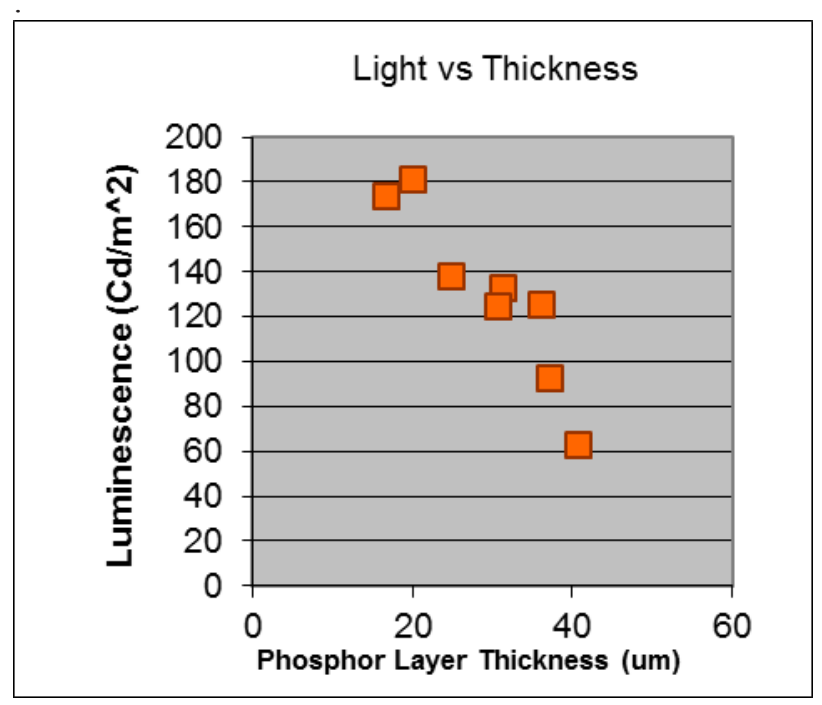

Figure.13: Lamp Brightness with Varying Phosphor Layer Thickness

An empirical equation can be obtained using this graph using graph fitting to find the relation between the thickness of phosphor layer and the Luminescence using excel as following

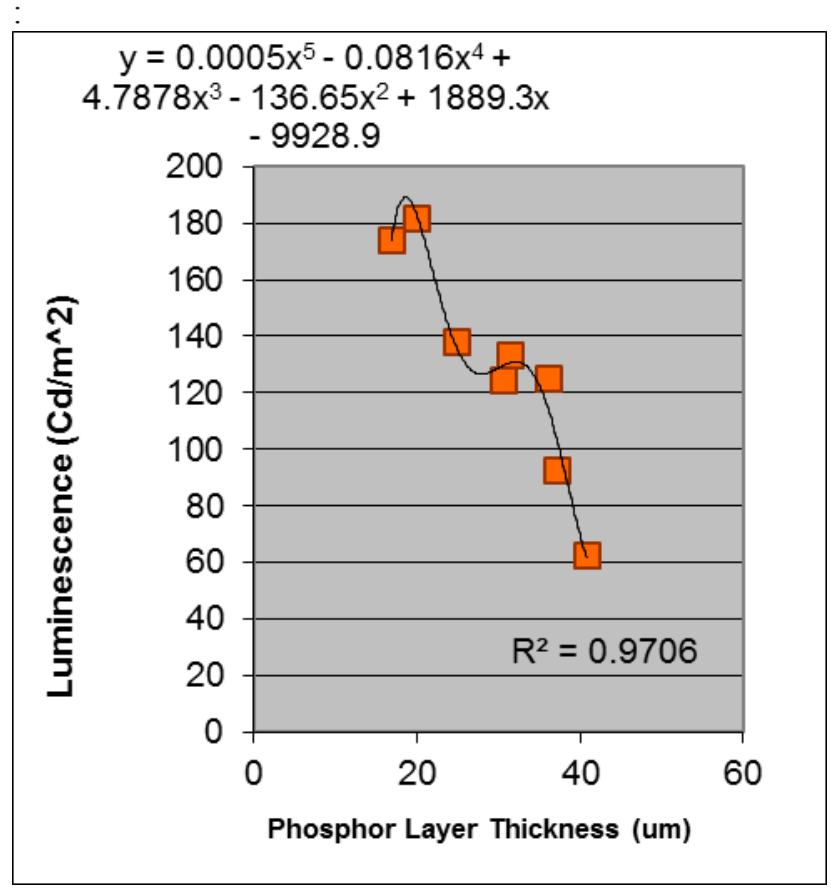

\section{Figure.14: Lamp Brightness with Varying Phosphor Layer Thickness curve fitting}

As shown the best fitting was polynomial with power 5 , where $\mathrm{R} 2$ value is 0.9706 which means there is a strength relation between the thickness and the Luminescence, where the relation between the phosphor thickness and the Luminescence is inversely relation as shown in the figure. 


\section{CONCLUSION}

Printing on a highly absorbent substrate material shows a unique set of practical challenges. In this report, water was supplied to a PEDOT: PSS substrate via Fibro DAT 1100 which is attached to a camera and a computer for capturing and analyzing coatings for the spread of a water droplet on the substrate surface. From these analyses, the main aim is to measure the contact angle between the droplet and the substrate surface which indicates the surface wettability. Some factors may affect the wettability of the surface such as varying the UV time and varying the hold time. The obtained results show that increasing these times will decrease the contact angle. A small contact angle indicates a favorable wettability. The second part in the experimental work which was used to measure the electrical resistance and transparency of the printed ink under different conditions showed that the values of electrical resistance increased with applying UV treatment. The electrical resistance of Ellenton is more than that for PEDOT at the same conditions. By changing the mesh ruling from (61-64) to (40-100), the value of resistance increases at the same conditions. For the transparency experiment the results present that Elentan ink has higher transparency than PEDOT ink.

A capacitor design was obtained in this research where the relation between the phosphor layer thicknesses was inversely proportion as shown in figures 36 and 37, so to increase the lamp output. For the future work, more than two inks can be used to select the most suitable type of inks. Also, the used machines for implementing the experimental work are not new so that it can be replaced by digital and automatic ones in the future.

\section{Authors}

J. G. Alotaibi obtained his $\mathrm{PhD}$ from Faculty of Health and Engineering at University of Southern Queensland, Australia. Before joining Public Authority for Applied Education and Training, he received his MSc from University of Bradford, United Kingdom.

- Ayedh Alajmi obtained his $\mathrm{PhD}$ from De Montfort University, United Kingdom. Before joining Public Authority for Applied Education and Training, he received his MSc from University of Brighton and university of Sussex, United Kingdom. . Currently, he is an Assistant Professor at the Public Authority for Applied Education and Training, Kuwait

Ahmad S M ALotaibi obtained his MSc of Advanced Manufacturing Technology/Technology Management from University of Portsmouth/ UK. he is engineer \& Projects Manager at Operation and maintenance Department, ministry of electricity and water/Kuwait .

\section{REFERENCES}

[1] J. Burgess, How Electroluminescent (EL) Wire Works, HowStuffWorks.com.. Accessed at 12/10/ 2014, 2010.

[2] J.A. Hart, S. A. Lenway, and T. Murtha, A History of Electroluminescent Displays, Second Draft.

Accessed at 14/10/2014, 1999.

[3] E. Kitazume, Organic electroluminescent device and the manufacturing method, US Patent No. 8002601 B2, 2011.

[4] B. Lee, and J. Lee, Printable flexible cholesteric capsule display with a fine resolution of RGB subpixels, Volume 11, Issue 6, November, 2011: 1389-1393.

[5] Y. Yuan, and T. R. Lee, Contact Angle and Wetting Properties, Department of Chemistry, University of Houston, 4800 Calhoun Road, Houston, TX 77204-5003, USA, 2013:127.

[6] H. Goo, Stephen Electrochromic inks including conducting polymer colloidal nanocomposites, devices including the electrochromic inks and methods of forming same. US, 2008.

[7] A. Adnan, Choil Printed Patterns Adhesion dependency on Contact Angle of Ink on Substrate, Korean Institute of Machinery and Materials, International Journal of Engineering \& Technology IJET-IJENS Vol:10 No:01 Daejeon, Korea 2010.

[8] M. Rehberger, A. O. Glasenapp, and J. Ortegren, VDP on Packaging - Elementary Velocity Study on Inkjet-printed Papers for Corrugated Board Production, 2010 :1-25.

[9] L. Shijian, and C.P. Wong, Effect of UV/ozone treatment on surface tension and adhesion in electronic packaging, Sch. of Mater. Sci. \& Eng., Georgia Inst. of Technol., Atlanta, GA, USA, Volume: 24 Issue:1,2002.

[10] H. Koyabashi, S. Kanbe, S Seki, A novel RGB multicolor light-emitting polymer display, Volumes 111-112, 2000:125128.

[11] F.J. Quites, G.C. Faria, and T.D.Z. Atvars, () White emission in polymer light-emitting diodes: Color composition by single-layer electroluminescence and external photoluminescence component, Material Letters, Volume 130, 2014: 65-67.

[12] M. K.Samokhvalov, R. R. Davydov, Determination of the Parameters of Phosphor Activators in Thin-Film Electroluminescent Capacitor Structures,ebesco academic journal, 2002 .

[13] K. Adrian, Luminescent Materials and Applications, John Wiley \& Sons-Technology \& Engineering, 2008:292. 